\title{
Alcance de la participación en la gestión de los consejos comunales
}

\author{
Olivar Morillo, Cira Elena*
}

\section{Resumen}

En el año 2006, surgen los Consejos Comunales como instancias de participación, articulación e integración entre los ciudadanos y las diversas organizaciones comunitarias; enmarcados en los preceptos constitucionales donde se concibe al ciudadano como sujeto activo en la gestión de los asuntos públicos. De estos planteamientos, se desprende como objetivo de investigación describir el alcance de la participación en la gestión de los Consejos Comunales de la parroquia Coquivacoa del Municipio Maracaibo. La metodología empleada fue de tipo descriptiva, con un diseño de campo; empleando como técnica de recolección de datos la entrevista. La población estuvo conformada por los consejos comunales ubicados en la Parroquia Coquivacoa. Los resultados evidencian que el alcance de la participación está estrechamente vinculado a la fase del ciclo comunal (gestión), pasando de una participación consultiva e instrumental, hasta llegar a niveles de decisión y ejecución. Se concluye que la participación en la gestión de dichos consejos, requiere de mayor protagonismo de los vecinos de estas comunidades donde están conformados, en pro de satisfacer las necesidades colectivas.

Palabras clave: Participación, gestión, consejos comunales.

\section{The Scope of Participation in Managing Communal Councils}

\section{Abstract}

In 2006, the Communal Councils arose as organizations for participation, coordination and integration among citizens and diverse community organizations, framed in constitutional precepts where the citizen is conceived as an active subject in managing public matters. Based on these proposals, the research topic arises of describing the scope of participation in managing Communal

\section{Recibido: 10-11-14 Aceptado: 15-01-15}

Magíster Scientarium en Gerencia Pública. Licenciada en Administración. Docente e Investigadora de la Facultad de Ciencias Económicas y Sociales, Universidad del Zulia. Centro de Estudios de la Empresa. Acreditada al Programa de Estímulo al Investigador (PEI) del Ministerio del Poder Popular para Ciencia, Tecnología e Innovación (MCTI). Maracaibo-Venezuela. E-mail: cira.olivar@gmail.com 
Councils in the Coquivacoa Parish, Maracaibo Municipality. Methodology was of the descriptive type with a field design, using the interview as a data collecting technique. The population consisted of the communal councils located in the Coquivacoa Parish. Results show that the scope of participation is narrowly connected to the phase of the communal cycle (management), passing from a consultative and instrumental participation until reaching levels of decision and execution. Conclusions are that participation in managing said councils requires greater protagonism from neighbors in the communities where they exist, in order to satisfy collective needs.

Key words: Participation, management, communal councils.

\section{Introducción}

En Venezuela -desde una visión formal- se puede señalar que con la aprobación de la Constitución de la República Bolivariana de Venezuela (CRBV, 2000), se consagra una nueva concepción de Estado y Sociedad en el país, enmarcada en un régimen democrático y participativo que sustituye la visión representativa de la democracia prevista en la constitución de 1961, caracterizada esta última, por un proceso de formulación de políticas públicas visto como responsabilidad exclusiva de las autoridades de turno, y por ende, de los partidos políticos que le acompaña, aspecto este que según López y Gamboa (2001:87), "deja a los ciudadanos indefensos ante posibles arbitrariedades o errores de los gobernantes".

Es importante acotar, la potestad de los Estados como organización jurídica de la sociedad, de definir y llevar a cabo políticas públicas que respondan a los intereses de esa sociedad que representan, es decir, concibiendo la política pública como el conjunto de decisiones o no decisiones (Vallés, 2000), orientadas a resolver o minimizar al máximo los conflictos propios del sistema democrático y los actores inmersos en el mismo. En palabras de Álvarez (1992), las políticas públicas son un modo de acción solo concebible razonablemente dentro del marco de un sistema político en donde el Estado tenga la facultad de regular, intervenir, impedir, compensar o reajustar los efectos indeseables del mercado y de sus propias acciones.

En virtud de lo expuesto, el Estado venezolano respondiendo a los preceptos contemplados en el marco constitucional (Artículos 62, 70) donde se concibe al ciudadano y sus diversas formas de organización como sujetos activos en la gestión de los asuntos públicos, ha desarrollado políticas orientadas a materializar esta intervención ciudadana en la planificación, ejecución, control y evaluación de la gestión pública.

En tal sentido, se comienza a gestar un proceso de participación en los procesos de toma de decisiones, manifestado en la intervención de las comunidades y grupos comunitarios organizados como sujetos clave en el diseño, organización, control y evaluación de políticas públicas que respondan a necesidades colectivas. Este proceso participativo encuentra sus raíces en la constitución pero además cuenta con una variedad de normativas 
legales ${ }^{1}$ que la rige y orienta, las cuales coinciden en definir el rol del ciudadano como activo y corresponsable en la solución de sus problemas.

En este escenario de impulso de la participación de los ciudadanos en los asuntos públicos, se dio inicio en el país por iniciativa del Ejecutivo Nacional, la conformación de los Consejos Comunales (CC), los cuales toman impulso a partir de la aprobación de su Ley en abril del 2006, modificada posteriormente, en el año 2009.

La modificación de esta normativa obedece a la necesidad de responder a algunos vacios presentes en su versión anterior, a la vez, de tratar de solventar trabas burocráticas que desde las instituciones públicas les son impuestas a los representantes de dichos consejos, como por ejemplo: respuestas tardías o no tramitación de solicitudes efectuadas, incumpliendo el artículo 51 de la CRBV; irrespeto al carácter de contralor de sus integrantes para hacer seguimiento a los proyectos ejecutados por instancias de gobierno; discrecionalidad o cambios de criterios por parte del funcionario público para efectuar un respetivo trámite ante la instancia respectiva, por mencionar algunos. En otras palabras, con ésta nueva ley, con rango orgánico, se establece la relación o coordinación que deben existir entre las instituciones públicas y los consejos comunales, así como la simplifica- ción de los trámites administrativos (Asamblea Nacional, 2009: Artículos 5660).

Del mismo modo, esta nueva ley de los CC profundiza el espíritu de horizontalidad e igualdad de autoridad entre sus integrantes y la comunidad, al establecer que su estructura está integrada por la Asamblea de ciudadanos(as), el colectivo de coordinación comunitaria y las unidades ejecutiva, administrativa y financiera comunitaria, y de contraloría social. Es importante acotar que el colectivo es un aspecto novedoso de coordinación y articulación del trabajo comunitario, y está integrado por los voceros y voceras de cada una de las unidades (Asamblea Nacional, 2009: Art. 19,24), lo cual denota la cooperación que debe existir entre sus integrantes, accionar consustanciado en valores y principios como la participación, corresponsabilidad, solidaridad, transparencia, rendición de cuentas, honestidad, bien común, colectivismo, eficacia, eficiencia, ética, responsabilidad social, control social, por mencionar algunos (Asamblea Nacional, 2009: Art. 3).

Los CC, en el marco legal que los regula, son definidos como instancias de participación, articulación e integración entre los ciudadanos y las diversas organizaciones comunitarias, movimientos sociales y populares, quienes de manera organizada ejercen el gobierno comunitario y la gestión directa de las políticas pú-

1 Ley Orgánica de las Comunas (2010), Ley Orgánica del Poder Popular (2010), Ley Orgánica del Sistema Económico Comunal (2010), Ley Orgánica de Contraloría Social (2010), Ley orgánica de Planificación Pública y Popular (2010) y Ley Orgánica para la Gestión Comunitaria de Competencias, servicios y otras atribuciones (2012). 
blicas y proyectos orientados a responder a las necesidades, potencialidades y aspiraciones de las comunidades (Asamblea Nacional, 2009: Art. 2).

Sin duda alguna, estas organizaciones comunitarias constituyen un espacio propicio para que las comunidades de manera organizada puedan intervenir en la gestión de sus problemas demandas y necesidades, por lo cual, este trabajo tiene como objetivo describir el alcance de la participación en la gestión de los consejos comunales de la Parroquia Coquivacoa del Municipio Maracaibo, estado Zulia. Para tal fin, se llevó a cabo una revisión sobre los aspectos conceptuales que encierra el proceso de participativo, así como una revisión normativa del objeto de estudio, y un acercamiento empírico de los consejos comunales de la Parroquia Coquivacoa del municipio Maracaibo.

La selección de los consejos comunales que funcionan en este marco geográfico, se fundamentó en un muestreo no probabilístico, intencional. Como criterios de selección de la muestra de estas organizaciones comunitarias, se tomaron en cuenta aquellos consejos comunales que: se le haya aprobado algún proyecto por algún órgano competente y que dichos proyectos se estén ejecutando y/o hayan sido ejecutados, esto con la finalidad de poder visualizar el ciclo comunal, donde se manifiesta la dinámica propia de la gestión de estas organizaciones comunitarias, así como la participación de los actores en el mismo, propósito de esta investigación.

Otro criterio de selección, fue que estos consejos comunales estuvieran constituidos desde el año 2006 y se ade- cuaron a las exigencias de la nueva ley aprobada en el 2009. Los consejos comunales que cumplen con estos criterios son Ocho (8), a saber: Alejandro Fuenmayor, La Merced del Valle, Norte Independiente, Ezequiel Zamora, Francisco de Miranda, 18 de Octubre Siglo XXI, Pueblo Aparte y El Renacer.

Para recolectar los datos en esta investigación se seleccionará como técnica, la entrevista; los sujetos informantes estarán representado por un (1) Vocero principal de la Unidad Administrativa y Financiera Comunitaria, un (1) Vocero principal por la Unidad de Contraloría Social y por un (1) por el Comité de trabajo responsable del proyecto ejecutado o en ejecución por el consejo comunal, para un total de veinticuatro (24) voceras y voceros entrevistados. Enmarcado en el propósito descriptivo del estudio, el mismo comprende un período que abarca la gestión comunal desde el año 2009 hasta la actualidad. Desde el punto de vista teórico la investigación se fundamenta en los postulados de autores como Cunill (1991), Fadda (1990), por citar algunos.

\section{Acepciones teóricas en torno a la participación}

La intervención de los ciudadanos en la actividad pública puede tomar diversidad de matices, en este particular Cunill (1991:41-46), sostiene que la participación puede expresarse en: Participación social: referida a los fenómenos de agrupación de los individuos en organizaciones a nivel de la sociedad civil para la defensa de sus intereses sociales; la Participación comunitaria, la cual supone una relación con el Estado pero que tiene sen- 
tido meramente de impulso asistencias de acciones que, en definitiva, son ejecutadas por los ciudadanos mismos y que, están vinculadas a su vida más inmediata, es decir, no se trata de una actividad pública sino de una actividad social.

Así mismo, se encuentra la Participación política: referida a los mecanismos tradicionales de ejercicio político como son las elecciones de representantes bajo la mediación de los partidos; y finalmente, la Participación ciudadana: concebida como un medio de socialización de la política en tanto supone generar nuevos espacios y mecanismos de articulación del Estado con los sujetos sociales; $y$ de fortalecimiento de la sociedad civil, constituido por una práctica social que asume la transferencia de funciones o decisiones que han permanecido tradicionalmente en manos del Estado o de la Administración Pública hacia la sociedad, es otras palabras, su finalidad es lograr "una redistribución del poder a favor de los sujetos sociales tradicionalmente excluidos de su ejercicio" (Cunill, 1991:38, 39).

En este sentido, la participación ciudadana tiene sentido en tanto justifica "crear nuevos mecanismos para que la Administración conozca mejor las necesidades de sus administrados, obtenga la colaboración de éstos en la ejecución de algunas tareas o bien, directamente, para que sustituya al Estado en la realización de determinadas funciones" (Cunill, 1991:43). En concordancia con esta visión, Dilla et al (1994:57), la conciben como "la capacidad que tiene el ciudadano común para involucrarse e incidir en los procesos de toma de decisiones, lo cual tiene un momento relevante en la participación electoral aunque no se limi- ta a ella". Es un proceso de construcción democrática y un medio para transformar las relaciones de poder, superando la brecha entre decisores y ejecutores.

Otra concepción de la participación es la expuesta por Fadda (1990: 46), quien parte del análisis de la teoría del conflicto para explicar las relaciones de poder de los grupos antagónicos, cuyos privilegios no pueden ser neutralizados por el sistema político. Desde esta perspectiva, la participación es asumida como "los esfuerzos organizados para incrementar el control sobre los recursos y las instituciones reguladoras en situaciones sociales dadas, por parte de grupos y movimientos de los hasta entonces excluidos de tal control".

La posición planteada por Fadda (1990), destaca varios elementos que se consideran relevantes para el análisis del significado de la participación: a) La consideración del antagonismo de grupos, unos dotados de poder y otros al margen de influir y controlar los recursos y las instituciones; b) Cambios en las relaciones de poder, al plantear la posibilidad de la organización de esfuerzos para incrementar el control sobre los recursos, dando cabida a diversas formas de participación; c) La participación puede darse desde abajo o desde arriba, dado que los esfuerzos organizados pueden venir de cualquiera de los dos extremos, siempre y cuando tenga como propósito, controlar a las instituciones reguladoras $y, d$ ) La referencia concreta a un contexto especifi$c o$, es decir, a una realidad en particular.

En vista a lo expuesto anteriormente, se asume la participación como la capacidad que tiene el ciudadano para incidir en la toma de decisión de las políticas 
públicas, en beneficio del colectivo, es decir, constituye el medio a través del cual la sociedad interviene en el proceso de planificación, ejecución y control sobre los asuntos de su interés, estableciendo así una nueva relación Estado-Sociedad Civil. Situación que requiere de la creación de mecanismos institucionalizados aceptables y reconocidos, que conlleven a la construcción de espacios para el encuentro entre los ciudadanos y el gobierno; no obstante, esto no representa una garantía para que realmente se haga efectiva la participación ciudadana, dado que en la práctica existen factores que la limitan; más allá de la voluntad política de los gobernantes, se encuentra: la apatía, desinformación, falta de tiempo, entre otros.

Una vez definida la participación es importante resaltar la importancia de este proceso para el desarrollo integral de las comunidades, así como su vinculación para el impulso de la transparencia y legitimidad de la gestión, sea esta municipal, regional o nacional. De igual manera, se resalta que la importancia de dicho proceso participativo no radica exclusivamente en su condición coadyuvante en la profundización de descentralización, sino, en constituirse en una herramienta mediante el cual los colectivos más débiles o vulnerables, los menos escuchados, pueden expresarse. En tal sentido, se busca que las comunidades participen y autogestión en los asuntos de interés colectivo, esto implica la autogestión social, en otras palabras, que los ciudadanos participen en la toma de decisión y gestión de los servicios públicos.

Es importante resaltar que espacios democráticos como los representa- dos por los consejos comunales, tienen limitantes que afrontar para su permanencia en el tiempo y que van más allá de la intervención gubernamental o inclusive de partidos políticos que deseen cooptarlos, y son aspectos intrínsecos en cada ciudadano como individuo con necesidades de múltiples naturaleza y que desea satisfacer. En palabras de Font (2001), se puede tener un escenario idílico donde existen ciudadanos muy preparados para participar, políticos muy predispuestos a esto y una vida política intensa y estimulante, por lo tanto, el patrón o interés de participar varía de una persona a otra, producto de las desigualdades entre clases y ocupaciones, recursos educativos o la capacidad de acceder y de comprender las informaciones políticas.

Esta situación, acota el autor antes citado, trae consigo algunos escepticismos o dudas en torno a la participación ciudadana, las cuales deben ser superadas en pro de la permanencia y legitimidad de los diversos mecanismos o instancias de participación en la gestión pública, estos escepticismos o dudas son:

a. Los ciudadanos, de hecho, no quieren participar; fundamentado en la idea de que los ciudadanos a la hora de la verdad no quieren implicarse realmente en el proceso complicado y fatigante en el que se acaba convirtiendo cualquier toma de decisiones públicas;

b. Los ciudadanos, cuando participan, lo hacen de una forma muy inconstante; argumentado en la variabilidad de asistencia de éstos ante las instancias participativas;

c. Los ciudadanos dispuestos a participar siempre son los mismos; origina- 
do por la asimetría de conocimientos e información por un lado, y de implicación y experiencia política por otro, ocasionan que los ciudadanos potencialmente capacitados o dispuestos a participar acaben constituyendo una pequeña minoría que siempre está presente, pero que difícilmente podemos aceptar que represente al conjunto de la ciudadanía;

d. Los ciudadanos que se movilizan y pretenden participar, muchas veces sólo se representan a ellos mismos y a sus intereses; se justifica en torno a la actuación de ciudadanos que se movilizan con la intención de defender intereses muy particulares y que por tanto no representan al conjunto de la ciudadanía.

Ahora bien, la participación más allá de las limitantes en cuanto a su práctica e incluso su postura teórica, representa para los ciudadanos, la oportunidad de intervenir en la gestión de asuntos colectivos, trabajar por el bien común y participar en toma de decisiones que atañen a la comunidad donde habitan, lo cual asumido con responsabilidad y capacidad ofrece canales para el mejoramiento de las condiciones de vida de las comunidades.

No obstante, la participación como estrategia reformista impulsada en la década de los noventa, ha tomado matices que inicialmente no la vinculaba con las comunidades como actores clave en los procesos participativos, sino que servicios públicos se iban transfiriendo a formas organizativas (privadas) su gestión, afianzando estrategias de privatización y minimizando en su máxima expresión, la figura del Estado, aspectos estos que responden a intereses de un modelo liberal o capitalista, donde el papel del Estado debe ser de regulador de las condiciones mínimas requeridas para lógica del mercado.

En este sentido, es importante mencionar lo expresado por Dussel (2006) en su trabajo 20 tesis de política, cuando refiere que la política tiene que ver con lo social, y que eso implica que desde el campo político se definen acciones para lograr la satisfacción de las reivindicaciones, atravesando otros campos como el económico, ecológico, cultural, entre otros. Del mismo modo, define claramente que "todo campo político es un ámbito atravesado por fuerzas, por sujetos singulares con voluntad, y con cierto poder" (Dussel, 2006:9); y asimismo, explica que "Todo campo tiene diversos sistemas. El campo político puede estar institucionalizado por un sistema liberal o socialista real, o por el sistema de participación creciente (como lo intenta la Revolución Bolivariana de Venezuela o la de Evo Morales en Bolivia) (Dussel, 2006:9). Por ende, el estudio de la participación, concebida como política del Estado venezolano, amerita una comprensión de los factores que convergen en la dinámica de dicha política pública.

\section{Alcance o características de la participación}

Partiendo de la visión de Cunill (1991), la participación o intervención de los particulares en actividades públicas en tanto portadores de determinados intereses sociales, se puede caracterizar en función de dos factores: 1) Los niveles y ámbitos en los que recaiga la participa- 
ción; y 2) El carácter de la intervención de los ciudadanos en las actividades y/o órganos públicos.

De este modo, la autora señala dos ámbitos: el político y de gestión, bajo los cuales se pueden configurar la Participación Ciudadana. El primero de ellos, el plano político, en donde se relaciona con la elaboración de las opciones o las decisiones de interés público, o sea con las actividades de gobierno. Entre los niveles de participación en este plano, se encuentran: la macro-participación: se refiere al ámbito propiamente político es decir, de las decisiones que comprometen el destino de la colectividad en su conjunto; la participación de nivel intermedio: su objeto son las políticas públicas de alcance sectorial o regional, que si bien afectan a amplios conglomerados no comprometen la sustancia del régimen político; y la participación de base o micro-participación: referida a las materias más cercanas al destino cotidiano de los individuos y los grupos pequeños. Las decisiones tomadas siguen siendo políticas, que si bien pueden ser influenciadas por procesos nacionales, éstas no afectan o impactan el devenir político de la comunidad global.

El segundo ámbito, es el plano de Gestión vinculado estrictamente con la generación de bienes y servicios públi$\cos$, y con las actividades de apoyo a éstos. En este ámbito la participación puede abarcar "desde el diagnóstico de la situación, la elaboración de las opciones o decisiones o la formulación de la política, programa o proyecto de desarrollo, pasando por la fase de ejecución y concluyendo en la fase de evaluación y control"
(Cunill, 1991:58). De esta manera, la participación puede distinguirse en:

- La participación consultiva y/o asesora: consiste en la expresión de opiniones o manifestación de conocimiento, pero sin que ello obligue al sujeto que adopta la decisión a un determinado curso de acción.

- La participación resolutiva y la participación fiscalizadora: implica la intervención en el curso de la actividad pública-participación decisoria- $y$, por tanto, ambas tienen un carácter obligante para la administración.

- La participación en la ejecución: supone que se toma en parte directamente en la realización de una actividad y/o en la prestación de un servicio.

Es importante destacar que estos tipos de participación, como bien lo expresa Cunill (1991), no poseen un carácter vinculante puede adoptar todas estas características más en el momento de implementar o ejecutar una política la participación no tiene cabida en este proceso, pues la misma queda en tarea de la administración, esta visión, nos lleva a referirnos a la dicotomía entre política y administración que plantean algunos autores, y que justifica que sean unos sujetos (analistas, políticos) quienes diseñen la política pero quien se encarga de su puesta en práctica es la burocracia o la administración.

Del mismo modo, Cunill (1991) para complementar los diferentes ámbitos dentro de los cuales se puede estudiar y comprender el fenómeno de la participación hace referencia a dos aspectos importantes: El primero es el carácter de 
los sujetos sociales intervinientes, donde se distingue la participación directa ejercida sin mediaciones, sea en forma individual o colectiva, y la participación indirecta dada por intervención de organizaciones que actúan en representación de intereses específicos. El segunda es el origen del impulso de la participación que puede darse a través de la acción ciudadana iniciada y controlada por los propios sujetos sociales con relación a propósitos que ellos determinan, y la implicación ciudadana la cual es iniciada y controlada por el Estado para mejorar y obtener apoyo para decisiones, programas o servicios.

En concordancia con la clasificación expuesta por Cunill en torno al proceso de participación Ochoa (1997:4), plantea que de acuerdo al papel de la gestión existen básicamente tres tipos de participación ciudadana: 1) La participación en la ejecución de las políticas públicas; 2) La participación consultiva, muy ligada a la opinión individual como consumidor, sin posibilidades de incidir en la formulación de políticas, dado el escenario "despolitizado" en el cual se desarroIla. Estos dos tipos de participación han tenido poco potencial para modificar la estructura de poder; y 3) La participación ciudadana resolutiva en función de una democratización que sirva de plataforma para la justicia social, se trata de un tipo de participación promovida desde la propia sociedad y que trasciende los objetivos de legitimación que se plantea el paradigma dominante de la gerencia pública, la cual hasta ahora no ha tenido posibilidades.
Esta tipología da cuenta de la manera como la participación de los ciudadanos se ha incluido en los programas y discursos de los gobiernos, así como en áreas de la gestión pública donde se concibe la incidencia de las comunidades, ello, rompiendo -por lo menos en el discurso- la concepción paternalista-populista del Estado, según la cual los ciudadanos, usuarios de los servicios, son parte externa al proceso de gestión y por lo tanto solo pueden ser receptores del servicio, dejando o delegando la responsabilidad exclusiva a la burocracia estatal y privada.

Otra posición es la manifestada por Mujica (2005:16-18), quién concibe la participación como el rol del ciudadano en cuanto partícipe y en cuanto usuario de las decisiones y gestiones asociadas a la implementación de acciones públicas; fundamentado en esta premisa, sostiene que la dicha participación puede presentar las diferentes características que marcan el alcance de la misma, a saber:

- La participación informativa: su objetivo es proveer información sobre el tema en cuestión; el flujo de información es unidireccional y no existe posibilidad de retroalimentación o negociación por parte de la ciudadanía, sin embargo, la información debe presentar una difusión adecuada, oportuna, completa y ampliamente accesible a la población.

- Participación consultiva: se plantea como opinión y control de parte de los interesados en el cumplimiento de los objetivos políticamente definidos por parte de la administración pública. 
- La participación decisoria: se orienta fundamentalmente a garantizar que las decisiones tomadas en la administración pública no se realicen en función de sus propios intereses sino de los intereses generales definidos políticamente; la participación de las personas y grupos es convocada en la perspectiva de influir respecto de un tema específico, a través de un proceso de negociación entre las partes involucradas a fin de establecer acuerdos de carácter vinculante. En este tipo, el sujeto que participa se piensa a sí mismo como co-productor y también velador en relación a la toma de decisiones de la administración pública.

- Participación como co-gestión/ejecutora: incluye la participación en el diagnóstico, la ejecución hasta la evaluación de las políticas públicas. Esta participación se asocia a la toma de decisiones en la gestión pública y la satisfacción de necesidades básicas. Esta modalidad considera a los beneficiarios como gestionadores de programas públicos para dar a respuesta a problemas que los afectan. Desde este punto de vista, puede pensarse en este tipo de participación en asuntos de carácter social, donde la ciudadanía pasa a ser parte de la ejecución de una determinada política. Supone también que exista liderazgo social e información para actuar como interlocutor de las agencias estatales.

- Participación instrumental: su propósito es la satisfacción de necesidades básicas y por ende la relación con la administración pública interesa sólo en cuanto permita acceder a estos recursos. Este tipo de participación instrumental utiliza la oferta tal cual viene predefinida desde los canales institucionales correspondientes. En este tipo de participación el sujeto actúa fundamentalmente como un usuario-cliente, y en cuanto tal no buscará incidir en la gestión pública en tanto obtenga los resultados deseados.

Una vez, explicados la diversidad de características que pueda presentar la participación y que define el alcance de la misma, en función del propósito del sujeto que la promueve o ejerce, es importante acotar que la participación promovida desde el Estado puede ser catalogada como manipulada, en el sentido que lleva implícito intereses propios del sistema de gobierno que la impulsa.

En tal sentido, los Consejos Comunales como mecanismo de participación ciudadana impulsado por el gobierno nacional en Venezuela, ha recibido críticas en torno a la vinculación gubernamental en su conformación, al considerárseles una instancia que responde a los intereses gubernamentales de consolidar sus principios socialistas y no en una medio eficiente a través del cual las comunidades solucionen sus problemas. A pesar de estas críticas, los consejos comunales se han convertido en el espacio propicio para que las comunidades detecten sus necesidades y diseñen en proyectos viables, las soluciones a las mismas, ello en conformidad con los principios democráticos y constitucionales de igualdad, corresponsabilidad, equidad y participación. 


\section{Características y alcance de la participación en la Gestión de los Consejos Comunales}

Para comprender la gestión de estas organizaciones comunitarias, así como el trabajo coordinado y cooperativo entre sus integrantes (voceros), se debe seguir un orden lógico de actuación, el cual es definido por la normativa como Ciclo Comunal (Asamblea Nacional, 2009: Art. 44), es decir, como el proceso para hacer efectiva la participación popular y la planificación participativa que responde a las necesidades comunitarias, a fin de contribuir al desarrollo de las potencialidades y capacidades de la comunidad. Su concreción se materializa a través de la realización de cinco fases, a saber: (Asamblea Nacional, 2009: Art. 45)

- Diagnóstico: esta fase caracteriza integralmente a las comunidades, se identifican las necesidades, las aspiraciones, los recursos, las potencialidades y las relaciones sociales propias de la localidad.

- Plan: es la fase que determina las acciones, programas y proyectos que atendiendo al diagnóstico, tiene como finalidad el desarrollo del bienestar integral de la comunidad.

- Presupuesto: esta fase comprende la determinación de los fondos, costos y recursos financieros y no financieros con los que cuenta y requiere la comunidad, destinados a la ejecución de las políticas, programas y proyectos establecidos en el plan comunitario de desarrollo integral.
- Ejecución: esta fase garantiza la concreción de las políticas, programas y proyectos en espacio y tiempo establecidos en el plan comunitario de desarrollo integral, garantizando la participación activa, consciente y solidaria de la comunidad.

- Contraloría social: esta fase es la acción permanente de prevención, vigilancia, supervisión, seguimiento, control y evaluación de las fases del ciclo comunal para la concreción del plan comunitario de desarrollo Integral y en general, sobre las acciones realizadas por el consejo comunal, ejercida articuladamente por los habitantes de la comunidad, la Asamblea de Ciudadanos, las organizaciones comunitarias y la Unidad de Contraloría Social del consejo comunal.

Ahora bien, con el fin de proceder a describir el alcance de la participación en la gestión de los consejos comunales, se procedió a indagar sobre cada una de las fases que conlleva el Ciclo Comunal, como expresión de las áreas clave de la gestión comunitaria como son: diagnóstico, planes, proyectos, presupuesto y contraloría social, aspectos explicados a continuación:

\section{a. Diagnóstico}

En esta fase, según los sujetos entrevistados, se pretende recopilar información de la comunidad en torno a sus potencialidades y debilidades, y posterior a ello, consultar en asamblea de ciudadanos a los vecinos en torno a la prioridad y posibles acciones de estas necesidades. Básicamente, se emplean instrumentos 
como los censos, el diagnóstico participativo y mesas de trabajo, a fin efectuar el levantamiento de información actual y precisa de la comunidad, por qué es de esta información que se extraen los datos requeridos para el proceso de planificación y elaboración de proyectos.

Ahora bien, en esta fase de la gestión se puede inferir que la participación se caracteriza y tiene un alcance netamente consultiva e informativa considerando los planteamientos de Cunill (1991) y Mujica (2005), cuando refieren que la participación consultiva se orienta a la posibilidad de expresar opiniones o conocimiento de los interesados en el cumplimiento de los objetivos de esta consulta, y la informativa, su finalidad es proveer información sobre el tema en cuestión de manera adecuada, oportuna, completa y ampliamente accesible.

\section{b. Planificación y presupuesto}

Esta fase, para los sujetos informantes, se ha desarrollado en los consejos comunales utilizando la información previa recopilada en la fase de diagnóstico, de allí su importancia de ser la primera fase del ciclo comunal. En referencia a las áreas en las cuales estos consejos comunales elaboran sus proyectos, se focalizan principalmente en el área de infraestructura, específicamente, la construcción de vivienda y sustitución de rancho por casa, rehabilitación de espacios físicos en escuelas, rehabilitación de áreas urbanas y casas comunales. No obstante, existen experiencias de proyectos socioproductivos ejecutados en estas comunidades, como es una fábrica de calzados y panadería comunitaria. Específi- camente se encuentran: Panaderías Comunitarias ( 1 en funcionamiento, 1 en ejecución la obra); fábrica de calzado (en funcionamiento); casas comunales y en ellas, espacios para consultorios Barrio Adentro; construcción del Comedor Escolar del Colegio Coquivacoa, así como recuperación de su infraestructura; reacondicionamiento del comedor de la UEN. Alejandro Fuenmayor; Sustitución y rehabilitación de viviendas, mediante la Gran Misión Vivienda Venezuela.

En relación a quienes son los sujetos que elaboran estos proyectos, los entrevistados manifestaron que la elaboración y presentación de planes y proyectos comunitarios es ejercida por los voceros y voceras, sin participación de los vecinos, pero al momento de su aprobación si se consulta con la comunidad, a través de la asamblea de ciudadanos como máxima instancia de decisión.

Otro aspecto resaltante en la planificación, fase clave en la gestión de los consejos comunales, está vinculada al apoyo o acompañamiento técnico que pueden recibir estas organizaciones al momento de elaborar sus planes y proyectos, encontrándose esta colaboración principalmente en el gobierno nacional a través de la Fundación para el Desarrollo y Promoción del Poder Comunal (FUNDACOMUNAL) ente adscrito al Ministerio del Poder Popular para la Participación y Protección Social, aunque en algunos momentos, según los sujetos informantes la colaboración recibida por el Frente Francisco de Miranda, la Escuela de Formación Popular y los lineamientos del Consejo Federal de Gobierno y la Sala de Batalla Social presente en la parroquia. 
Esto concuerda con lo expresado por Cunill (1991), cuando señala que las comunidades organizadas, ineludiblemente, serán en un futuro o presente no tan inmediato, las constructoras de su propia realidad, ello valiéndose de herramientas vitales como la planificación para idear soluciones efectivas a las necesidades y aspiraciones comunitarias. Del mismo modo, esta dinámica sociopolítica que engloba los CC, da cuenta de una nueva relación entre el Estado y los ciudadanos (as) que impulsará nuevos mecanismos que permitan a la administración pública conocer mejor las necesidades de sus administrados y trabajar en conjunto con ellos en la solución de las mismas.

No obstante, es necesario destacar que en materia presupuestaria los CC presentan limitantes por qué dependen exclusivamente del financiamiento de recursos públicos, así lo manifestaron los sujetos informantes quienes identifican como principales fuentes de financiamiento a FUNDACOMUNAL, el Consejo Federal de Gobierno, el Servicio Autónomo Fondo Nacional de los Consejos Comunales (SAFONAC), pero la capacidad de respuesta de estas instancia no es tan rápida como la deseada por ellos, originando molestias en la comunidad por este tipo de demoras y trabas burocráticas.

Tomando en cuenta lo expresado, el rasgo que caracteriza a esta fase del ciclo comunal, es la participación decisoria según lo expresado por Mujica (2005), en el entendido que las decisiones tomadas no se realicen en función de sus propios intereses sino de los intereses generales definidos políticamente; a través de un proceso de negociación entre los habitantes de la comunidad.

Del mismo modo, presenta rasgos de una intervención de tipo ejecutora ( $\mathrm{Cu}$ nill, 1991), no sólo por tener los voceros(as) la competencia legal de elaborar los planes y proyectos, sino porque a través de estas herramientas se detectan las áreas prioritarias para la inversión de los recursos públicos; a la vez, se presentan rasgos instrumentales (Mujica, 2005), observándose el ciudadano como receptor de beneficios, esto debido a que el propósito de esta planificación es satisfacer las necesidades sociales de los ciudadanos, pero no en promover e impulsar organizaciones de la economía social, como cooperativas, empresas de producción social, por mencionar algunos, actores clave para el desarrollo sostenible de las comunidades. Esto se evidencio en los proyectos elaborados y ejecutados en el área y la poca promoción de actividades de autogestión que le genere ingresos a estas organizaciones comunitarias, tratando de minimizar en algunas áreas la dependencia con el Estado.

\section{c. Ejecución}

La participación en esta fase, es limitada exclusivamente a los voceros y voceras, quienes asumen la responsabilidad del cumplimiento del proyecto, en el tiempo establecido y con optimización de los recursos financiados, así lo expresaron los sujetos informantes. Así mismos, comentan que los vecinos solo participan en la consulta efectuadas a través de la Asamblea de Ciudadanos para establecer el orden de los proyectos, su aproba- 
ción a fin ser presentado ante el respectivo ente de financiamiento.

La característica esencial en esta fase, es la consultiva e instrumental (Cunill, 1991 y Mujica, 2005), debido a la poca capacidad de decisión o influencia de los consejos comunales en la aprobación de los recursos, aunado pues dependen de otras instancias de financiamiento, básicamente gubernamentales. Del mismo modo, quienes participan directamente en la ejecución de los proyectos, son los voceros(as), con poca intervención y seguimiento de los vecinos de la comunidad, manteniéndose así el principio de representatividad.

Por otro lado, una vez aprobados los recursos, quienes participan en el ejecútese del proyecto son los voceros de los CC, llevando a cabo las actividades inherentes al mismo, por ejemplo, si se requiere personal para la ejecución de proyecto, los voceros en función de la información disponible del diagnóstico comunitario, seleccionan los vecinos con las habilidades solicitadas, sean de su ámbito geográfico o cercano al mismo basado, en este último caso trabajando en conjunto con los CC aledaños a su comunidad.

En consecuencia, se puede visualizar rasgos característicos de la intervención ejecutora en esta fase, tal como lo expresan (Cunill, 1991 y Mujica, 2005), cuando sostienen que este tipo de intervención se caracteriza porque el ciudadano toma parte directa en la realización de una actividad y/o en la prestación de un servicio, o bien desde el carácter social, pasa a ser parte de la ejecución de una determinada política.

\section{d. Contraloría social}

Esta fase se encuentra referida al proceso de prevención, vigilancia, seguimiento, control y evaluación de la gestión comunitaria como de las instituciones tanto públicas como privadas que funcionan en el ámbito geográfico de competencia de los consejos comunales.

Para los sujetos informantes, esta fase ha sido tímida, pues la vigilancia y el control se han llevado a cabo en la ejecución de los proyectos comunitarios, pero muy poco se ha evaluado la gestión del propio consejo comunal y en ningún momento la de instituciones públicas o privadas. Como se menciono anteriormente, cuando se manejo la contraloría como una forma de participación, se enfatizo en que los sujetos contralores básicamente son los voceros de esta unidad en la estructura del consejo comunal, y el vocero comprometido en la ejecución del proyecto.

El seguimiento se enfoca en los procesos de Rendición de Cuentas y Declaración Jurada de Bienes por parte de los voceros de la unidad administrativa y financiera comunitaria, como instrumentos que garantiza la transparencia y responsabilidad de la gestión comunitaria. En consecuencia, con ello, se retoma la esencia de este proceso de control como el espacio propicio para dar seguimiento a la actuación de las instituciones públicas, en este caso comunitaria, a fin de orientar su acción a valores como la transparencia, rendición de cuentas y sensibilidad social, esto quiere decir, al manejo oportuno y eficiente de los recursos. 
En síntesis, la intervención de los ciudadanos en el control y seguimiento de la gestión comunitaria, se lleva a cabo como un proceso interno de los CC, poco se promueve la contraloría ejercida por los vecinos, dándose de este modo un ejercicio limitado de este mecanismo de participación; se maneja una participación consultiva, ya que solo se involucra a las comunidades en el proceso de rendición de cuentas a fin de informarle lo realizado en la gestión comunitaria. Esto contradice el espíritu de este proceso y de esta forma de participación expuesto por Ochoa (2006), cuando expresa que la misma implica la defensa y resguardo de los intereses colectivos y no de los individuales, sobre todo cuando se hace referencia al espacio público, a la gestión de los asuntos públicos, los fines perseguidos deben ser colectivos, es decir, el de todos los ciudadanos por igual.

Se puede finalizar este objetivo, retomando la concepción de los CC como instancias de participación, articulación e integración entre las diversas organizaciones comunitarias, grupos sociales y los ciudadanos (as), y asumiendo que la concepción participativa de los CC se ubica siguiendo a Cunill (1991) y Mujica (2005), en el plano de la gestión pública, en vista de la posibilidad que tienen las comunidades de intervenir en la toma de decisión con carácter vinculante; así como en la implementación, control y evaluación de las mismas. Constituyéndose las comunidades organizadas en constructoras de su propia realidad, a través de la determinación de proyectos $y$, en general de las acciones públicas orientadas a responder a sus necesidades y aspiraciones.

\section{Reflexiones finales}

Se puede aseverar que el alcance de la participación promovida en la gestión de los consejos comunales, depende de la fase del ciclo comunal analizada, pasando de una participación consultiva e informativa para el diagnóstico hasta tener rasgos de una participación ejecutora y de cogestión en la fase ejecución de los proyectos; se hace hincapié en la cercanía de la visión instrumental en la gestión de los consejos comunales, al darle un carácter más asistencial (búsqueda de soluciones a deficiencias en servicios o infraestructuras públicas) que a la promoción de una actividad socioproductiva enmarcada en una economía social, que consolide el desarrollo integral de estas comunidades.

Para finalizar, se precisa la necesidad de impulsar la articulación de los gobiernos municipales con estas organizaciones, a fin del adecuado acompañamiento y orientación técnico-político que les permita la cohesión de los planes comunales, con los municipales, regionales y nacionales, en pro de optimizar la inversión pública pero también promover que estos planes y proyectos propicien el desarrollo comunitario y sustentabilidad de la gestión comunitaria, y no, a la reproducción de la dependencia del financiamiento gubernamental por partes de las organizaciones comunitarias o populares.

\section{Referencias bibliográficas}

\section{Álvarez, Ángel (1992). Análisis de Políticas} Públicas. Serie temas de Coyuntura en Gestión Pública. Venezuela. Cen- 
tro Latinoamericano de Administración para el Desarrollo (CLAD), pp.39.

Asamblea Nacional Constituyente (2000). Constitución de la República Bolivariana de Venezuela. Gaceta Oficial Extraordinaria $N^{\circ} 5.453$. Venezuela.

Asamblea Nacional de la República Bolivariana de Venezuela (2009). Ley Orgánica de los Consejos Comunales. Gaceta Oficial $\mathrm{N}^{\circ}$ 39.335. Venezuela.

Asamblea Nacional de la República Bolivariana de Venezuela (2010). Ley Orgánica de las Comunas. Gaceta Oficial N ${ }^{\circ}$ 6.011. Venezuela.

Asamblea Nacional de la República Bolivariana de Venezuela (2010). Ley Orgánica del Sistema Económico Comunal. Gaceta Oficial № 6.011. Venezuela.

Asamblea Nacional de la República Bolivariana de Venezuela (2010). Ley Orgánica de Contraloría Social. Gaceta Oficial $N^{\circ} 6.011$. Venezuela.

Asamblea Nacional de la República Bolivariana de Venezuela (2010). Ley Orgánica del Poder Popular. Gaceta Oficial Extraordinaria $N^{\circ} 6.011$. Venezuela.

Asamblea Nacional de la República Bolivariana de Venezuela (2010). Ley Orgánica de Planificación Pública y Popular. Gaceta Oficial Extraordinaria No 6.011. Venezuela.

Asamblea Nacional de la República Bolivariana de Venezuela (2012). Ley Orgánica para la Gestión Comunitaria de competencias, servicios y otras atribuciones. Gaceta Oficial Extraordinaria $N^{\circ} 6.079$. Venezuela.

Cunill, Nuria (1991). Participación Ciudadana. Dilemas y perspectivas para la democratización de los Estados Latinoamericanos. Venezuela. Centro Latinoamericano de Administración para el Desarrollo (CLAD), pp. 262.
Dilla, Haroldo; González, Gerardo; Vicentelli, Ana (1994). Participación Popular y Desarrollo en los Municipios Cubanos. Venezuela, Fondo Editorial Tropykos, pp. 173.

Dussel, Enrique (2006). 20 Tesis de Política. México, Siglo XXI editores. Disponible en: http://www.afyl.org/txt/LibrosNovedades/20-Tesis.pdf, fecha de consulta: 03/05/2014. pp. 174.

Fadda, Giulietta (1990). La Participación como Encuentro. Discurso Político y Praxis Urbana. Venezuela. Fondo Editorial Acta Científica Venezolana. Universidad Central de Venezuela, pp. 282.

Font, Joan (2001). Decisiones públicas y ciudadanía: Nuevos mecanismos e instrumentos de participación ciudadana. España, Ayuntamiento de Alicante. Disponible en: http://www. alicante.es/documentos/p_ciudadana/2_decisiones_publicas.pdf, 03/05/ 2006, pp. 6-30.

López Valladares, Mirtha; Gamboa Cáceres, Teresa (2001). Democracia y Participación en los Municipios Venezolanos: Bases Constitucionales, Legales y Teóricas. Revista Ciencias de Gobierno. Año 5. No 10. Venezuela, Instituto Zuliano de Estudios, Políticos, Económicos y Sociales (IZEPES), Gobernación del Estado Zulia, pp. 77-99.

Mujica, Pedro (2005). La participación ciudadana en relación a la Gestión Pública. Chile. Corporación Participa, pp. 25.

Ochoa Henríquez, Haydee (1997). Emergencia del Paradigma de la Gerencia Pública en Venezuela. II Congreso Internacional del CLAD sobre la Reforma del Estado y de la Administración Pública, Isla de Margarita, Venezuela, 14 - 18 de octubre 1997. Disponible en: 
http://siare.clad.org/fulltext/0032012. pdf, fecha de consulta: 03/05/2006, pp. 16.

Ochoa Henríquez, Haydee (2006). Contraloría Social en la Gestión Pública. Charla a la VIII Corte de Maestría en Gerencia
Pública. Facultad de Ciencias Económicas y Sociales de la Universidad del Zulia. Fecha: 05/04/2006. Maracaibo, Venezuela.

Vallés, Joseph (2000). Ciencia Política: Una introducción. España. Editorial Ariel. Barcelona, pp.442. 